\title{
Drosophila TFIIA directs cooperative DNA binding with TBP and mediates transcriptional activation
}

\author{
Kyoko Yokomori, Martin P. Zeidler, ${ }^{1}$ Jin-Long Chen, C. Peter Verrijzer, Marek Mlodzik, ${ }^{1}$ \\ and Robert Tjian
}

Howard Hughes Medical Institute, Department of Molecular and Cell Biology, University of California, Berkeley, California 94720-3202 USA; ${ }^{1}$ European Molecular Biology Laboratory, 69012 Heidelberg, Germany

\begin{abstract}
Drosophila transcription factor IIA (TFIIA) is composed of three subunits $(30,20$, and $14 \mathrm{kD})$ that function during initiation of transcription. We reported previously the characterization of cDNAs that encode a precursor (dTFIIA-L) of the Drosophila TFIIA 30- and 20-kD subunits. In the absence of the smallest subunit, dTFIIA-S $(14 \mathrm{kD})$, the unprocessed large subunit failed to exhibit any detectable promoter binding or transcriptional activity. Here, we report the molecular cloning and expression of dTFIIA-S, which has allowed the assembly of holo-dTFIIA (dTFIIA-L/S). Subunit interaction studies indicate that dTFIIA-S binds to an amino-terminal domain of dTFIIA-L, which likely corresponds to the endogenous $30-\mathrm{kD}$ processed species. In addition, both dTFIIA-S and the carboxy-terminal domain of dTFIIA-L, which corresponds to the 20-kD species, independently interact weakly with the TATA-binding protein (TBP). In contrast, the holo-dTFIIA (L/S) binds TBP with high affinity. The dTFIIA-L/S complex also binds cooperatively with TBP to TATA box DNA sequences, generating an extended DNase footprint pattern. The reconstituted holo-dTFIIA is able to stimulate basal transcription of several core promoter templates. Interestingly, dTFIIA-L/S is also able to significantly enhance transcriptional activation by upstream transcription factors including Sp1, VP16, and NTF-1. These results suggest that dTFIIA is a multifunctional transcription factor capable of influencing DNA binding as well as interactions with the basal machinery, thereby enhancing activator-dependent transcription.
\end{abstract}

[Key Words: Drosophila; TFIIA; DNA binding; transcriptional activation ]

Received June 30, 1994; revised version accepted July 29, 1994.

Early studies of the eukaryotic transcriptional machinery responsible for directing mRNA synthesis suggested that there were two functionally distinct and nonoverlapping classes of transcription factors: basal components that assemble at core elements of the promoter common to most RNA polymerase II transcription units and promoter-selective factors that bind to specific arrays of DNA recognition sites unique to each gene. However, recent advances in characterizing the structure and function of transcription factors required to assemble activated initiation complexes suggest that this classic model may be an oversimplification. Instead, it is now clear that in addition to basal factors and promoter-specific activators, there are also coactivators that are not required for basal transcription but, rather, help mediate activation and perhaps repression (Pugh nd Tjian 1990; Meisterernst and Roeder 1991; Meisterernst et al. 1991; Inostroza et al. 1992; Merino et al. 1993). However, the division of labor between basal factors and coactivators and/or adaptors is poorly defined and some transcription factors may contribute to both processes. Thus, it is probable that components assembled during initiation of transcription are multifunctional with the capacity to influence different steps during the transcription cycle.

Although recent studies suggest the existence of such multifaceted transcription factors, the identity and biochemical properties of these putative "crossover" transcription factors have remained elusive. One likely candidate for a functionally versatile component of the eukaryotic transcription complex is transcription factor IIA (TFIIA). A number of studies suggest that TFIIA is not required for transcription at all promoters but that in some situations it is able to significantly enhance basal levels of transcription, possibly by counteracting the effects of inhibitors (Samuels et al. 1982; Reinberg et al. 1987; Van Dyke et al. 1988; Merino et al. 1993). In addition, several recent studies report the potential involvement of TFIIA in mediating activator-dependent transcription (Wang et al. 1992; Chi and Carey 1993; Ma et al. 1993; Lieberman and Berk 1994). Interestingly, during chromatographic fractionation of Drosophila nuclear extracts, TFIIA was found associated with TFIID, which contains TATA-binding protein (TBP) and TBP-associated factors (TAFs) (Yokomori et al. 1993). Because TFIIA 
has also been documented to interact directly with the TBP subunit of the TFIID complex (Reinberg et al. 1987; Ranish and Hahn 1991; Cortes et al. 1992; Ranish et al. 1992), TFIIA could also be considered a TAF with potential coactivator function. These intriguing observations prompted us to more fully characterize the biochemical properties of TFIIA.

Our previous work revealed that Drosophila TFIIA (dTFIIA) consists of three subunits $(30,20$, and $14 \mathrm{kD})$ that can be purified in association with the TBP-TAF complex (Yokomori et al. 1993). In contrast to the core TAFs, however, the subunits of TFIIA can be readily eluted with $0.5 \mathrm{~m}$ potassium chloride. Molecular cloning and characterization of the large subunit (dTFIIA-L) revealed that both the 30 - and $20-\mathrm{kD}$ species are probably derived from a single precursor product of $48 \mathrm{kD}$ encoded by dTFIIA-L that is the homolog of the large subunit of yeast TFIIA (TOAl; Ranish et al. 1992). Microsequencing of the endogenous subunits and analysis of dTFIIA-L deletion mutants as well as antibody cross-reactivity experiments map the $30-\mathrm{kD}$ subunit to the amino-terminal half of the precursor protein, whereas the $20-\mathrm{kD}$ species appears to derive from the carboxy-terminal portion of the molecule. The smallest (14 kD) subunit of dTFIIA remained to be characterized and was predicted to be the homolog of the yeast small subunit, TOA2 (Ranish et al. 1992). Although the unprocessed product (dTFIIA-L) was able to interact weakly with TBP directly, we were unable to study the potential DNA-binding or coactivator properties of dTFIIA, because the $48-\mathrm{kD}$ precursor protein failed to function in vitro in the absence of the 14$\mathrm{kD}$ small subunit. Thus, the contributions of TFIIA to either initiation complex formation or transcriptional enhancement remained to be defined.

Here, we report the isolation, molecular cloning, and expression of the remaining small subunit, dTFIIA-S. First, we have assembled a "holo-TFIIA" (dTFIIA-L/S) containing the recombinant unprocessed $48-\mathrm{kD}$ dTFIIA-L subunit together with the small subunit dTFIIA-S. We then tested the ability of reconstituted dTFIIA-L/S to interact directly with TBP both in the absence and presence of the TATA box DNA. Finally, we have determined the ability of dTFIIA-L/S to enhance both basal and activator-dependent transcription in vitro. Our results suggest that dTFIIA is a multifunctional transcription factor that contributes to the formation of a stable protein-DNA complex at the core promoter and helps mediate activation of transcription.

\section{Results}

Identification, molecular cloning, and expression of dTFIIA-S

To isolate cDNAs encoding the small subunit (dTFIIA$\mathrm{S}$ ), we have purified the $14-\mathrm{kD}$ polypeptide by preparative immunoaffinity chromatography followed by amino acid sequence analysis of peptide fragments. At the same time, we identified several cDNA clones from Drosophila that contained an open reading frame with similar amino acid sequence to the small subunit of yeast TFIIA. These dTFIIA-S cDNAs were isolated in a systematic search for transcription units in the vicinity of enhancer detector insertion F125 (M.P. Zeidler and M. Mlodzik, unpubl.). One genomic fragment flanking the insertion site hybridized to a set of small cDNAs in both Drosophila embryonic and eye disc libraries. Subsequent analysis of these clones revealed that the transcript is ubiquitously expressed and encodes a polypeptide with homology to the yeast TOA2 protein (Ranish et al. 1992). All cDNAs analyzed contained an open reading frame preceded by a stop codon and polyadenylation signals (Fig. 1A). When comparing the amino acid sequences determined by microsequencing of the purified Drosophila 14-kD species to sequences deduced from these cDNA clones, we found two peptides that corresponded to the open reading frame (Fig.1A, underlined sequences), confirming the identity of these cDNAs as encoding dTFIIA$\mathrm{S}$. The entire deduced amino acid sequence revealed $>40 \%$ identity and $60 \%$ similarity to yeast TOA2 (Ranish et al. 1992), 60\% similarity to the rice homolog of TFIIA (DDBJ: D15390, submitted by Y. Minobe, National Institute of Agrobiological Resources, Japan), and $87 \%$ similarity to the small subunit of human TFIIA /D. Reinberg, pers. comm.) (Fig. 1B) . Our dTFIIA-S sequence predicts a protein with a calculated molecular mass of $12.2 \mathrm{kD}$ and an estimated isoelectric point (PI) of 8.9.

To characterize further the product of dTFIIA-S, we have used both Escherichia coli and baculovirus expression systems to overproduce recombinant protein. First, the bacterially expressed product was used to generate polyclonal antibodies. Western blot analysis confirmed that the antibodies specifically recognized a $14-\mathrm{kD}$ protein that was coimmunoprecipitated by antibodies against dTFIIA-L (Fig. 1C, lane 1). In addition, isolation of the TFIID complex from Drosophila extracts with anti-dTAF ${ }_{\mathrm{II}} 250$ also revealed the presence of a $14-\mathrm{kD}$ protein that was recognized by anti-dTFIIA-S (Fig. 1C, lane 2). As expected, the baculovirus-expressed recombinant dTFIIA-S comigrated with the endogenous $14-\mathrm{kD}$ protein, confirming that the clone we have obtained encodes the full-length protein (Fig. 1C, lane 3).

\section{Protein-protein interactions between TBP and subunits of dTFIIA}

Previously, we had shown that the large, unprocessed subunit dTFIIA-L is capable of binding weakly to TBP in coimmunoprecipitation experiments. However, such an incomplete TFIIA species lacking the small subunit was unable to form a stable complex with TBP bound to DNA, as determined by mobility retardation assays (DeJong and Roeder 1993; Ma et al. 1993; K. Yokomori, unpubl.). With the small subunit now available in recombinant form, we have returned to investigate the interaction of dTFIIA and TBP. Baculovirus-expressed recombinant TBP was first immobilized on protein $A$ beads via a monoclonal antibody specific to TBP. Control beads contained antibody alone (Fig. 2, even numbered lanes). The beads were then incubated with radio- 


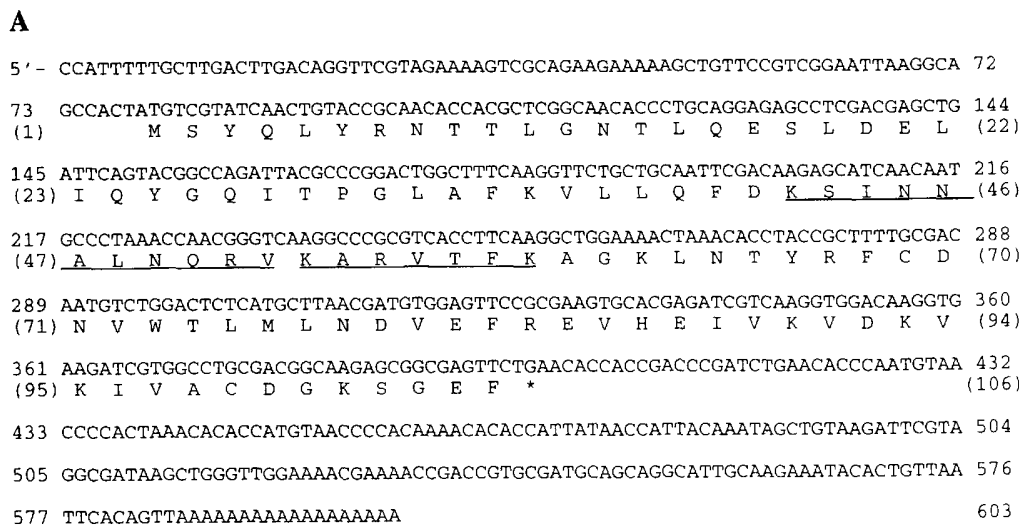

$\mathbf{B}$

RICe 1 NKEKSGVSSSPPLLLSLRQQADSRSPSGKKSRAEQGRGSCSLGA

HUMan 1 MAYQLYRNTTLGNSLQESLDELIQSQQITPQLALQVLLQFDKAINAALAQRVRNRVNNFR Dros 1 MSYQLYRNTTLGNTLQESLDELIQYGQITPGLAFKVLLQFDKSINNALNQRVKARVTFKA $|*||| *|*| 1 *|* *||||||* *||* *||||| * * *|* * * *| * \mid$ Yeast 1 MAVPGYYELYRRSTIGNSLVDALDTLISDGRIEASLAMRVLETFDKVVAETLKDNTQSKLTVK Rice 45 RIAHGHLRALPESTIGMCLTETLDEMVSSGTLSPELAIQVLVQFDKSMTEALENQVKSKVSIK

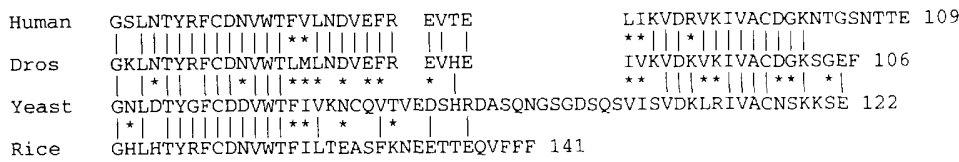

C
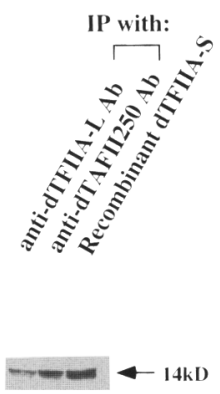

$1 \quad 2 \quad 3$

Western blot with anti-dTFIIA-S Ah dTFIIA-S protein migrates during electrophoresis with a mobility identical to the endogenous $14-\mathrm{kD}$ subunit of TFIIA. The endogenous TFIIA complex (lane 1) and TBP-TAF complex (lane 2) were immunopurified by anti-dTFIIA-L and anti-dTAF 250 antibodies (Ab), respectively, and compared with baculovirus-expressed recombinant dTFIIA-S (lane 3) by SDS-PAGE. The endogenous 14-kD subunit and recombinant TFIIA-S were visualized by Western blot using anti-dTFIIA-S Ab (arrow).

labeled ${ }^{35} \mathrm{~S}$ in vitro-translated dTFIIA-L, dTFIIA-S, or cotranslated dTFIIA-L/S proteins (Fig. 2). In the presence of both subunits of TFIIA, we now observe highly efficient binding to TBP $(>10 \%$ of input; Fig. $2 \mathrm{~A}$, lanes 4,5$)$. The ratio of the large to small subunits bound on the beads is $\sim 1: 1$ based on PhosphorImager analysis. In contrast, neither the large subunit $\mathrm{L}$ nor the small subunit $\mathrm{S}$ alone interacted efficiently with TBP $(<1 \%$ ) (Fig. 2, lanes 6-9; lanes 10-13 represent the longer exposure of lanes $6-9$. These results suggest that the presence of both large and small subunits of TFIIA greatly enhances the binding to TBP.

The equimolar ratio of TFIIA-L to TFIIA-S bound to TBP suggests that these two subunits form a specific complex. To analyze these potential protein-protein interactions, we used an HA-tagged TFIIA-L (Yokomori et al. 1993 |, which was immobilized on beads and tested for binding to ${ }^{35}$ S-labeled TFIIA-L or TFIIA-S in the absence of TBP (Fig. 2). As expected, HA-dTFIIA-L interacted with dTFIIA-S efficiently $>10 \%$ of the input was bound; cf. lanes 3 and 17). We also found that dTFIIA-L can interact with itself (lanes 14,15$)$. This might reflect the natural interaction between the processed parts of TFIIA-L in the cell. However, we have not yet been able to locate precisely proteolitic cleavage sites and show the assembly of the processed subunits. dTFIIA-S is capable of interacting with both TBP and the large subunit TFIIA-L, and both subunits of TFIIA are able to form a stable complex with each other in the absence of TBP. Taken together with the TBP-binding results, our data suggest that specific TFIIA complex formation greatly stabilizes the interaction with TBP even in the absence of DNA.

Because the endogenous dTFIIA- $L$ is cleaved into two fragments ( 30 and $20 \mathrm{kD}$ ), we were interested in mapping the domains within dTFIIA-L that are responsible for the interactions with TBP and dTFIIA-S. We used two artificial ${ }^{35} \mathrm{~S}$-labeled fragments of the large subunit, termed $\mathrm{LN}$ and $\mathrm{LC}$, which roughly correspond to the endogenous fragments 30 and $20 \mathrm{kD}$, respectively (Yokomori et al. 1993). Figure 2B shows that TBP interacts with LC (lanes 1-6), which contains a 100-amino-acid conserved region at the carboxyl terminus of the protein (Yokomori et al. 1993). HA-dTFIIA-S, on the other hand, interacted only with LN, the amino-terminal half of the large subunit, which contains a 60-amino-acid conserved region at the amino terminus (Yokomori et al. 1993). An internally initiated translation product of LN that utilizes the second methionine and lacks the first 39 amino acids within the conserved region (lane 7 , indicated by *) failed 
A

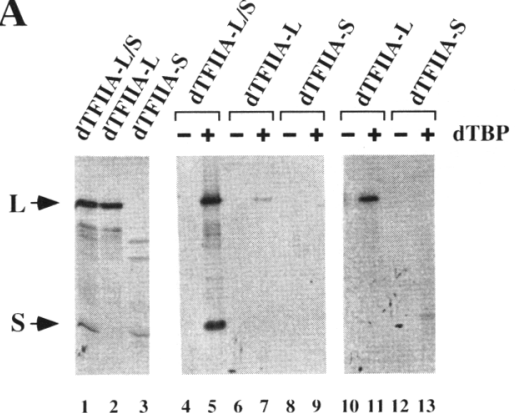

(S)
(L)

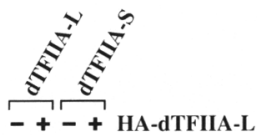

-+-+ HA-dTFIIA-I

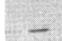

$-$

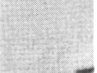

14151617

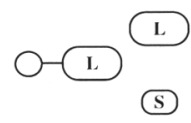

B
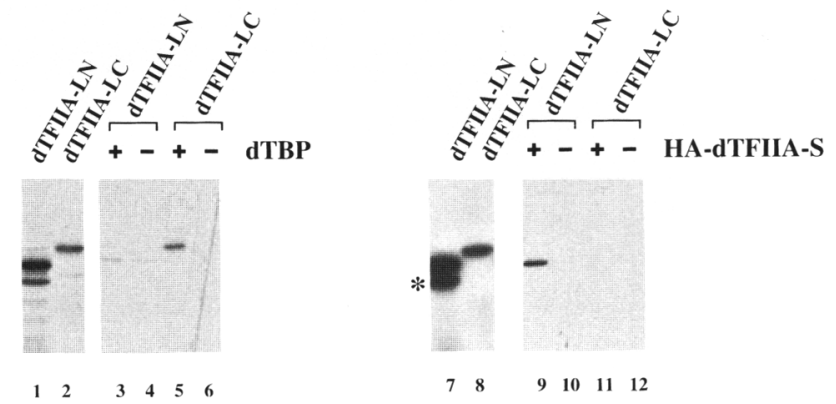

$\mathrm{O}-\stackrel{\mathrm{dTBP}}{\stackrel{\mathrm{LN}}{\mathrm{LC}}}$

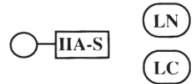

Figure 2. dTFIIA-S enhances TBP binding of dTFIIA-L by interacting with both dTFIIA-L and TBP. (A) Coimmunoprecipitation of ${ }^{35}$ S-labeled dTFIIA-L/S, dTFIIA-L or dTFIIA-S with dTBP (lanes 4-13) or with HA-dTFIIA-L (lanes 14-17). Recombinant dTFIIA-L and dTFIIA-S were transcribed and translated either together $(\mathrm{L} / \mathrm{S})$ or separately in the presence of $\left[{ }^{35} \mathrm{~S}\right]$-methionine in the TNT T7 polymerase-rabbit reticulocyte lysate system (Promega). As shown in the schematic diagrams (bottom), equivalent amounts of ${ }^{35}$ S-labeled proteins were incubated with antibodies specific for either dTBP or HA peptide on protein A beads in the presence (lanes 5-17, odd-numbered lanes) or in the absence (lanes 4-16, even-numbered lanes) of dTBP or HA-dTFIIA-L. Five percent of the input is shown in lanes 1-3. All lanes are from the same exposure except lanes 10-13, which represent a longer exposure of lanes $6-9$ to reveal the weak signal of ${ }^{35}$ S-labeled dTFIIA-S (lane 13). (B) TBP binds to the carboxy-terminal half of dTFIIA-L, whereas dTFIIA-S binds to the amino-terminal half. Radiolabeled dTFIIA-LN (amino acid 1-222) and dTFIIA-LC (229-366) were synthesized by in vitro translation and incubated with beads containing dTBP or HA-dTFIIA-S as shown in the schematic diagrams. Control beads contained antibody against dTBP or HA alone (lanes 4,6,10,12). Lanes 1,2, 7, and 8 show $5 \%$ of each input. A truncated LN product initiated from the second methionine, lacking the first 39 amino acids, is indicated by an asterisk $(\star)$.

to bind HA-dTFIIA-S (lane 9). Thus, the first 39 amino acids of dTFIIA-L are important for specific interactions with dTFIIA-S. These results indicate that both ends of the dTFIIA-L are required for assembly of a stable TBPTFIIA complex. Furthermore, these results suggest that the endogenous $30-\mathrm{kD}$ amino-terminal processed form of dTFIIA-L is responsible for binding to the small subunit, whereas the $20-\mathrm{kD}$ carboxy-terminal product of the precursor large subunit is responsible for TBP interactions.

\section{Recombinant purified dTFIIA-L and dTFIIA-S can form a stable complex with TBP on DNA}

It has been shown previously that the endogenous TFIIA is able to make a stable complex with TBP on TATA box DNA in mobility retardation assays (Reinberg et al. 1987; Ranish and Hahn 1991; Cortes et al. 1992; Yokomori et al. 1993) and exhibit a specific pattern of DNase protection upstream of the TATA box region in the presence of TBP (Buratowski et al. 1989). Therefore, we attempted to reconstitute recombinant holo-dTFIIA (L/S) in vitro and use it to test its DNA-binding activity in the presence of TBP. First, bacterially produced dTFIIA-L and DTFIIA-S were purified, denatured with urea, and then allowed to renature together (Fig. 3A). As controls, dTFIIA-L and dTFIIA-S were separately subjected to the same denaturation and renaturation treatment. Interestingly, bacterially expressed dTFIIA-S is highly insoluble by itself but appeared to be substantially more soluble in the presence of dTFIIA-L. These preparations of recom- binant proteins were then used in mobility retardation assays (Fig. 3B). TBP alone produced a very weak protein-DNA complex (lane 1, as indicated). The addition of endogenous dTFIIA $(0.5 \mathrm{M}$ eluate $)$ resulted in a prominent, slower migrating complex (Fig. 3B, lane 2, as indicated). The addition of recombinant dTFIIA-L/S also produced a strong complex that migrated at an almost similar but slightly faster position than the endogenous TBP-TFIIA complex (lane 3). As expected, neither dTFIIA-L alone nor dTFIIA-S alone in the presence of TBP was capable of giving a shift (lanes 4,5). Similarly, the dTFIIA holocomplex was unable to bind DNA in the absence of TBP (lane 6). These results suggest that the cleavage of dTFIIA-L into the endogenous $30 \mathrm{kD}$ and 20 $\mathrm{kD}$ subunits is apparently not required to form a holocomplex capable of binding to TBP and forming a stable DNA complex on the TATA box template. However, we note that the recombinant unprocessed dTFIIA-L/S-TBP complex migrates slightly faster than that of the endogenous dTFIIA-TBP complex (Fig. 3B, lanes 2,3), which may be the result of the lack of processing of dTFIIA-L.

We then performed DNase I footprint protection using a DNA fragment containing the adenovirus major late promoter (nucleotides -116 to +61 ) labeled at the $5^{\prime}$ end of the noncoding strand. First, we found that $\sim 20 \mathrm{ng}$ of TBP alone was required to protect the TATA box on the template from DNase digestion, producing a characteristic footprint between position -19 and -36 centered around the TATA box, with an enhanced cleavage site at -16 . In contrast, in the presence of increasing 
A

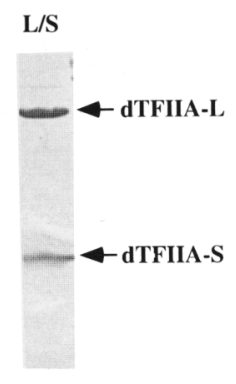

B
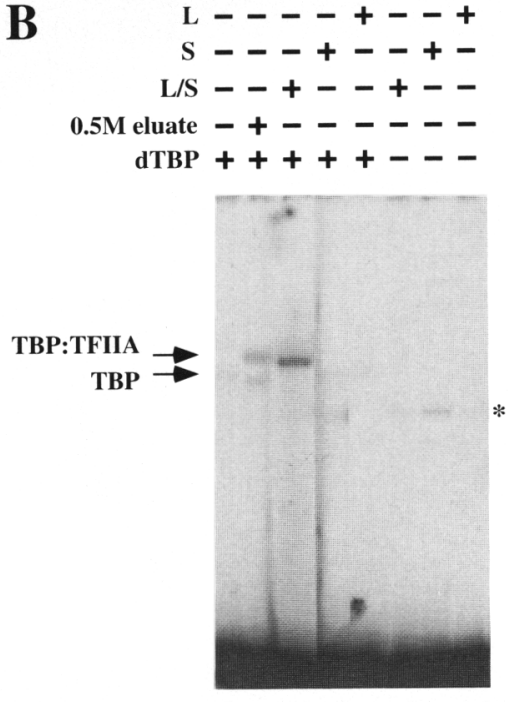

$\begin{array}{llllllll}1 & 2 & 3 & 4 & 5 & 6 & 7 & 8\end{array}$

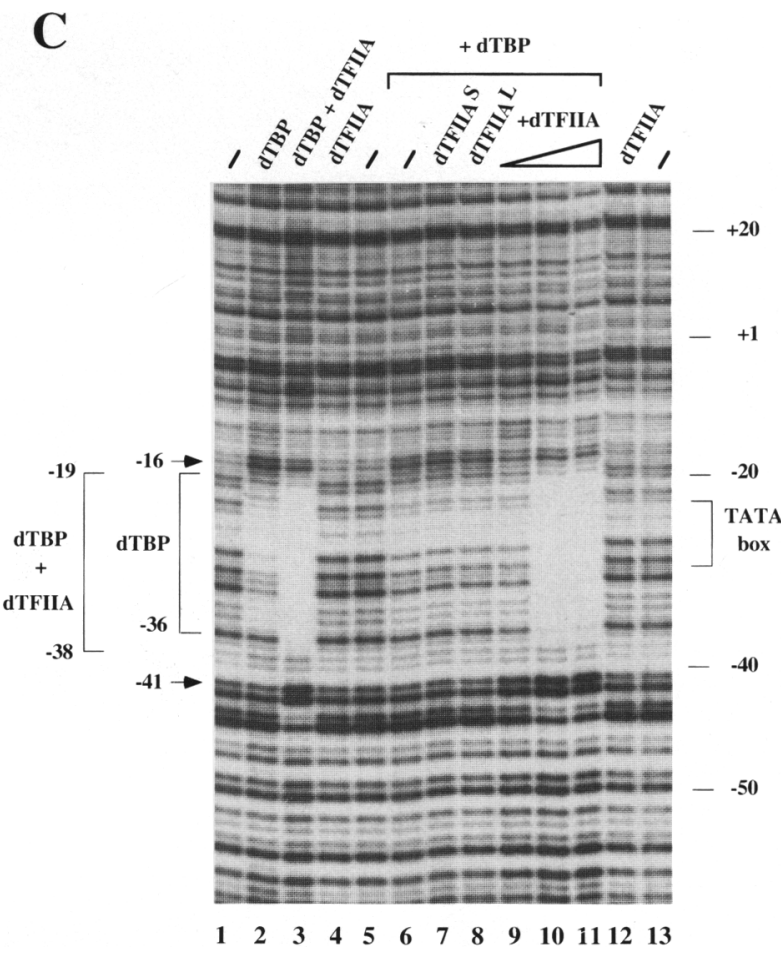

Figure 3. Holo-dTFIIA (L/S) enhances TBP binding to TATA box-containing DNA. (A) Coomassie staining of holo-dTFIIA (L/S) expressed in bacteria, denatured, and renatured together. $(B)$ A complex of dTFIIA-L/S with TBP on TATA box-containing DNA forms a shifted complex. The relative positions of a dTBP-DNA complex and TBP-TFIIA-DNA complex are indicated. As controls, similar amounts of $\mathrm{L}$ and $\mathrm{S}$ denatured and renatured separately were also tested for DNA binding. Nonspecific binding by a bacterial contaminant is indicated by the asterisk $\left({ }^{*}\right) .(C)$ DNase I footprinting analyses of dTBP and recombinant holo-dTFIIA at the AdML promoter. A DNA fragment containing AdML promoter sequences was radiolabeled at the $5^{\prime}$ end of the nontranscribed strand and incubated in the presence of the indicated proteins. The protein-DNA complexes were partially digested with DNase I and analyzed on a $6 \%$ polyacrylamide gel. The binding reactions contained: no protein (lanes 1,5,13); $20 \mathrm{ng}$ of dTBP alone (lane 2) or $20 \mathrm{ng}$ of dTBP plus $25 \mathrm{ng}$ of holo-TFIIA (lane 3); $25 \mathrm{ng}$ TFIIA alone (lanes 4,12). Lanes 6-11 all contained $1.5 \mathrm{ng}$ of dTBP either in the absence of TFIIA (lane 6) or in the presence of $40 \mathrm{ng}$ of TFIIA-S (lane 7); $40 \mathrm{ng}$ of TFIIA-L (lane 8); 2, 12.5, or $25 \mathrm{ng}$ of holo-TFIIA (lanes 9,10,11, respectively). Numbers indicate the positions in the AdML promoter relative to the start site $(+1)$. The protected regions are indicated by brackets.

amounts of recombinant dTFIIA-L/S, a much smaller amount (1.5 ng) of TBP was needed to completely protect the TATA box region. Therefore, TFIIA has a $>10$-fold stabilizing effect on DNA-binding of TBP. In addition, we observed that TBP plus holo-dTFIIA extended the region of protection upstream to position -38 with a novel enhanced cleavage site at -41 . Thus, the addition of dTFIIA-L/S to TBP greatly stabilizes binding of the TBP-TFIIA complex to the TATA box region. In contrast, the addition of recombinant dTFIIA-L or dTFIIA-S subunit alone failed either to stabilize TBP binding or to produce an extended footprint. As expected, addition of holo-dTFIIA-L/S in the absence of dTBP had no effect on the DNase footprint pattern. These results suggest that holo-dTFIIA binds cooperatively with TBP to the TATA box and that the dTFIIA subunits may directly contact the DNA when associated with TBP.

\section{Recombinant dTFIIA stimulates basal transcription}

Because holo-TFIIA (L/S) appeared to be functional in
DNA binding when complexed to TBP, we then tested its ability to function in transcription. To test the activity of recombinant dTFIIA in transcription, we have used a fractionated in vitro transcription system that requires exogenously added TFIID and TFIIA. We have tested basal transcription from several different templates, including a hybrid of the core promoter elements from the adenovirus major late gene fused to the TdT initiator (AdML-TdT) and the core promoter elements from adenovirus E4 and E1B genes (see diagram in Fig. 4). The in vitro transcription products were detected by primer extension. On the AdML-TdT template, we observed a low level of basal transcription in reconstituted reactions lacking endogenous TFIIA fraction (Fig. 4, lane 1). Similar low levels of basal transcription were seen when either recombinant purified dTFIIA-S or dTFIIA-L alone was added to these reactions (Fig. 4, lanes 4-7). In marked contrast, when increasing amounts of TFIIA-L/S were added to the reactions, transcription was stimulated up to 10-fold (cf. lanes 1 and 3). We noted consistently that basal transcription was most prominently 
Figure 4. Recombinant holo-dTFIIA (L/S) stimulates basal transcription directed by TFIID. Schematic diagram of the three DNA templates used here and in Fig. 5 are shown, which include the AdML TATA box fused to TdT initiator, and E4 and E1B promoters. HeLa nuclear extract was fractionated by phosphocellulose chromatography, and transcription was reconstituted with the various fractions including the $0.5 \mathrm{M}$ fraction (PC0.5) containing all of the general factors except TFIIA and TFIID and $1.0 \mathrm{M}$ fraction (PCl.0) containing TFIID. Comparable amounts of $L / S, S$, and $L$ were added (ranging from 10-50 ng). The numbers at the top of each lane correspond to the range of concentrations tested. The levels of transcription were quantitated by PhosphorImager (Molecular Dynamics).

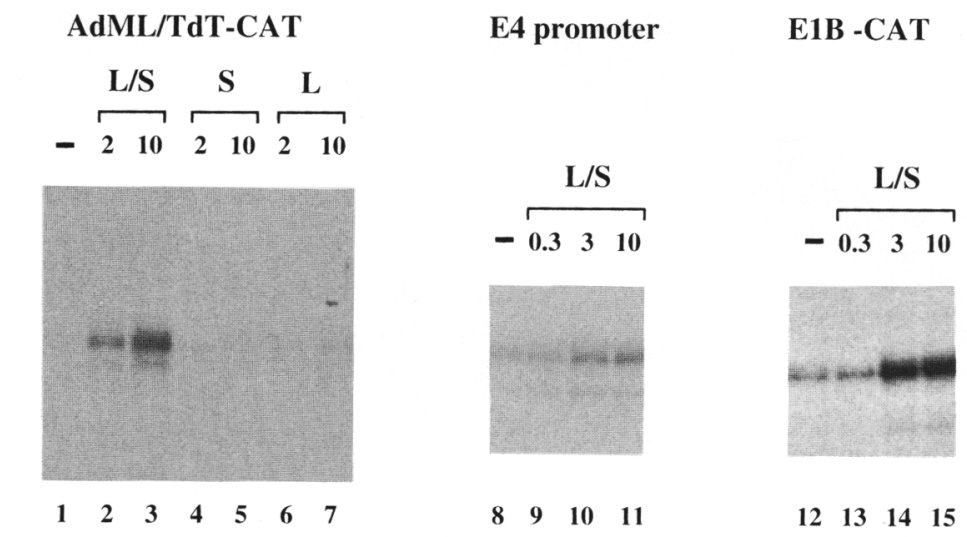

AdML/TdT-CAT

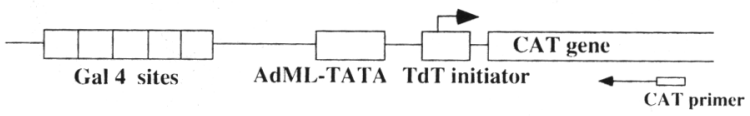

E4 promoter

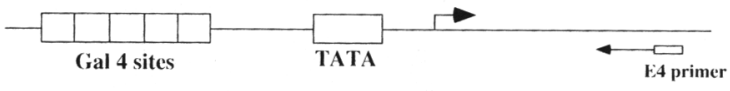

E1B-CAT

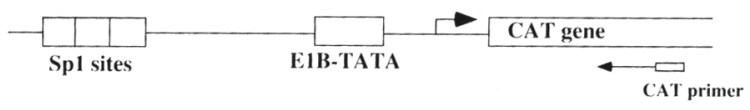

stimulated only at the higher concentrations of recombinant holo-dTFIIA. Similar enhancement of basal transcription by dTFIIA-L/S was observed with the E1B promoter (Fig. 4, lanes 12-15), and a somewhat more modest stimulation was seen with the E4 promoter (Fig. 4, lanes $8-11$ ). These results suggest that at least for these three core promoters, the presence of the dTFIIA holocomplex is able to facilitate some step during transcription and thereby stimulate the TFIID-dependent basal activity.

\section{Recombinant dTFIIA enhances transcriptional activation}

Although TFIIA has traditionally been considered a basal factor, a number of recent observations suggest that perhaps TFIIA also plays an important role in activated transcription (Wang et al. 1992; Chi and Carey 1993; Ma et al. 1993; Lieberman and Berk 1994). Consequently, we were prompted to study its effects, if any, on activation by a variety of upstream transcription factors bearing different types of activation domains, including NTF-1, Spl, VP16, and Zta. First, we tested the hybrid activator, Gal4-NTF-1 using an AdML-TdT promoter template bearing multiple upstream Gal4-binding sites (see diagram in Fig. 4). As expected, in the absence of the activator, the presence of dTFIIA-L/S stimulated basal transcription, whereas dTFIIA-S or dTFIIA-L alone did not (Fig. 5A, lanes 1-5). Interestingly, when Gal4-NTF was added to these same reactions, we observed a dramatic enhancement of transcription in the presence of dTFIIAL/S (Fig. 5A, cf. lane 6 with lanes 7 and 8 ) and no stim- ulation with either subunit alone (cf. lane 6 with lanes 9-12). The greatest enhancement was observed when relatively low levels of dTFIIA-L/S were used. Amounts of dTFIIA that failed to significantly enhance basal transcription had a maximal effect on activator-stimulated transcription. We estimate that in the absence of dTFIIA, Gal4-NTF stimulates transcription 9-fold above basal (Fig. 5A, cf. lanes 1 and 6$)$, L/S (0.3) had no effect on basal transcription (lane 2), whereas activation was stimulated 3.2 times more than the activation without TFIIA (cf. lanes 6 and 71, thus resulting in 29-fold activation above the basal. The GAL4 DNA-binding domain by itself does not stimulate transcription (data not shown).

These initial observations led us to perform several additional experiments with low levels of added dTFIIA$\mathrm{L} / \mathrm{S}$ so that minimal stimulation of basal transcription would occur. Under these conditions, basal transcription was rather low and could only be detected with longer exposures (data not shown) or by PhosphorImager quantitation (Fig. 5A,B). With low levels of dTFIIA-L/S, we found a marked enhancement of activated transcription not only with Gal4-NTF but also with Gal4-VP16 (N/ C), Gal4-VP16 (C) as well as Spl (Fig. 5B,C). For example, we observed an average of fivefold enhancement of GAL4-NTF-dependent transcription with no detectable stimulation of basal transcription by TFIIA. In the case of GAL4-VP16 activators, the effect of TFIIA was even more dramatic. GAL4-VP16C exhibited virtually no activation (less than 2-fold) in the absence of TFIIA (cf. lanes 1, 9, and 11), whereas the presence of TFIIA stimulated the activation up to 10 -fold (Fig. 5B) without af- 


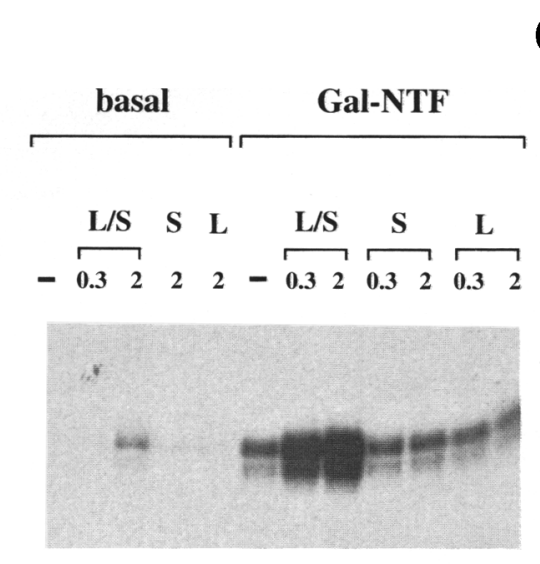

$\begin{array}{llllllllllll}1 & 2 & 3 & 4 & 5 & 6 & 7 & 8 & 9 & 10 & 11 & 12\end{array}$

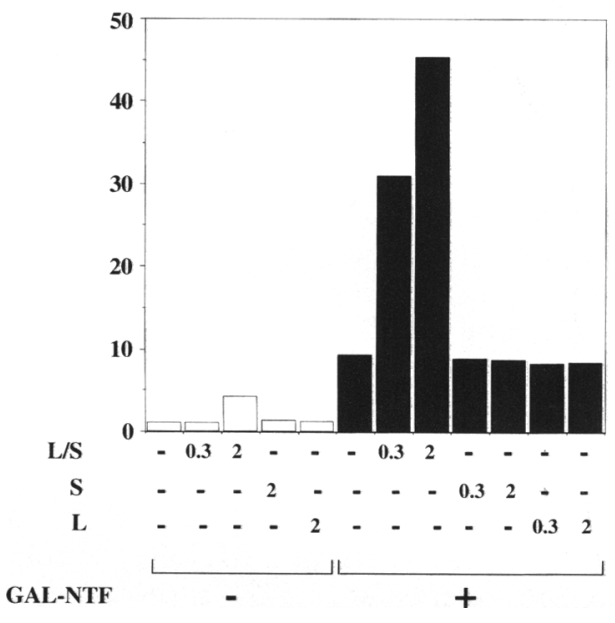

B

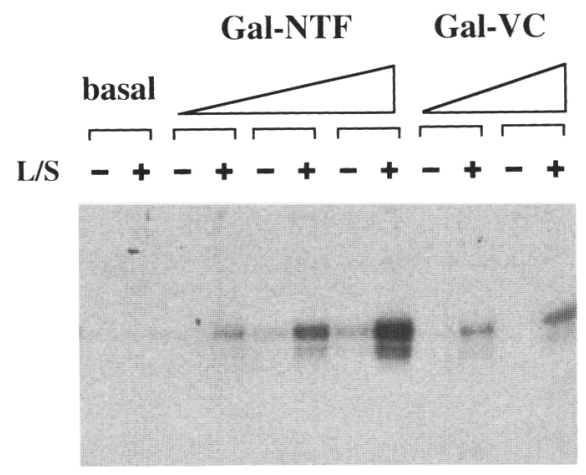

$\begin{array}{llllllllllll}1 & 2 & 3 & 4 & 5 & 6 & 7 & 8 & 9 & 10 & 11 & 12\end{array}$

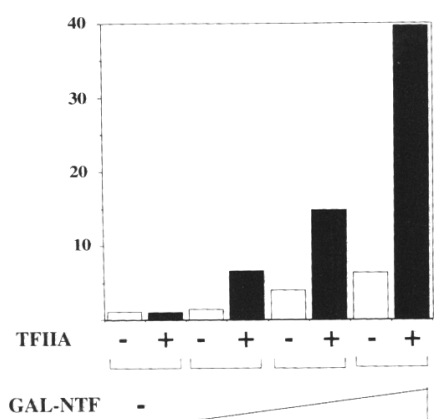

GAL-NTF

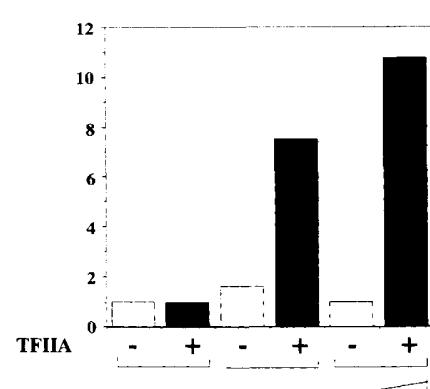

GAL.VC
AdML-TdT-CAT
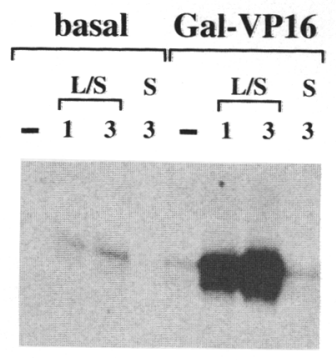

$\begin{array}{llllllll}1 & 2 & 3 & 4 & 5 & 6 & 7 & 8\end{array}$

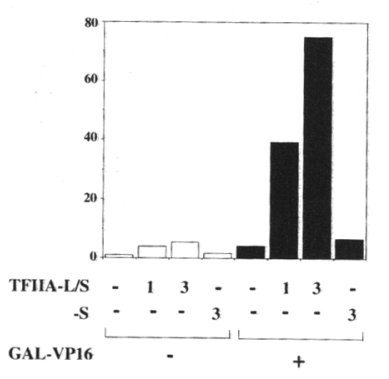

\section{E1B-CAT}

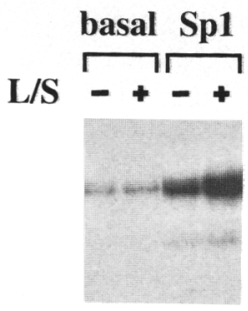

$\begin{array}{llll}9 & 10 & 11 & 12\end{array}$

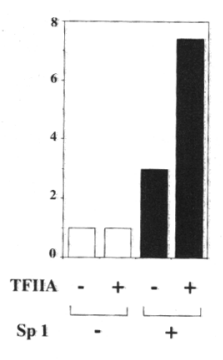

D

\section{$\mathrm{Z}_{7} \mathrm{E}$ 4TCAT}

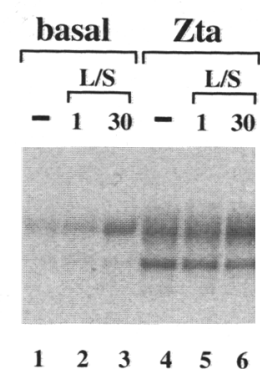

Figure 5. Holo-dTFIIA stimulates transcriptional activation. (A) GAL-NTF activation on AdML-TdT promoter is stimulated by L/S but not by S or L alone. (Lanes 1-5) The effect of $\mathrm{L} / \mathrm{S}, \mathrm{S}$, or $\mathrm{L}$ on basal transcription (cf. lane 1 with other lanes). The amounts of dTFIIA proteins (indicated at the top of each lane) are the same concentrations as in Fig. 4. (Lanes 6-12) Transcription in the presence of GAL-NTF. Stimulation of activation by TFIIA should be assessed by comparing the signal between reactions containing the same amounts of TFIIA, such as lanes 1 and 6 /both without TFIIA|, lanes 2 and 7, and lanes 3 and 8 . In all cases, the products were quantitated by PhosphorImager analysis. All values were relative to the basal transcription without TFIIA, which was designated as 1 . (B) Low levels of TFIIA (L/S) stimulated activation by GAL-NTF and GAL-VP16C without affecting basal transcription. The level of activation by different concentrations of GAL-NTF and GAL-VP16C (as indicated at top) were compared with samples containing TFIIA (even-numbered lanes) and samples lacking TFIIA (odd numbered lanes). At these concentrations of TFIIA, no stim ulation of basal transcription was seen (cf. lanes 1 and 2). (C) TFIIA greatly stimulates activation by GAL-VP16 and to a lesser extent Spl. GAL4-VP16 activation was tested on AdML-TdT, Spl on E1B-CAT template. (Open bars) Transcription in the absence of activators; (solid bars) transcription in the presence of a fixed concentration of an activator. $(D) \mathrm{Zta}$ activation does not require TFIIA and is not affected by the addition of TFIIA. An E4TCAT template DNA with seven $\mathrm{Z}$ ta-binding sites was used. (Lanes 1-3) Transcription in the absence of $\mathrm{Zta}$; (lanes 4-6) transcription in the presence of $\mathrm{Zta}$. Two different amounts of TFUA were tested as indicated. 
fecting the basal transcription. Similarly, the modest 4-fold activation by GAL4-VP16 without TFIIA was stimulated 19 times more in the presence of TFIIA /cf. lanes 5 and 7), resulting in 75-fold activation above basal without TFIIA (cf. lanes 1 and 7), whereas the basal transcription was elevated only 5 -fold with the same amount of TFIIA (cf. lanes 1 and 3) (Fig. 5C). These results taken together suggest that holo-dTFIIA is able to significantly accentuate the activation properties of some enhancer factors in reconstituted transcription reactions.

A recent report suggested that trans-activation by the viral protein $\mathrm{Zta}$ is dependent on TFIIA as a potential cofactor (Lieberman and Berk 1994). We therefore also tested the ability of our recombinant holo-dTFIIA-L/S to mediate activation by $\mathrm{Zta}$ from the $\mathrm{Z}_{7} \mathrm{E} 4 \mathrm{TCAT}$ template (Lieberman and Berk 1994) under our in vitro transcription conditions. Surprisingly, in contrast to Gal4-NTF, VP16, and Spl, we were unable to observe any stimulation of transcription by dTFIIA-L/S of the viral activator Zta, which by itself was sufficient to give high levels of activation (Fig. 5D). Presently, we are unable to reconcile our in vitro results with those reported previously (Chi and Carey 1993; Lieberman and Berk 1994). One possibility is that Drosophila TFIIA does not function on Zta activation. Alternatively, it is possible that under our assay conditions, the effect of TFIIA on the initial step of activation may be masked. Finally, the unprocessed holo-TFIIA may fail to mediate $\mathrm{Zta}$ activation.

\section{Discussion}

Previously, we reported cloning the large subunit of Drosophila TFIIA (TFIIA-L) (Yokomori et al. 1993). Here, we report the molecular cloning of the small subunit dTFIIA-S and the assembly of an active holo-TFIIA. The small subunit dTFIIA-S binds to the conserved aminoterminal region of dTFIIA-L, whereas the carboxy-terminal domain of the large subunit is involved in TBP binding. In vivo, TFIIA-L from both human and Drosophila apparently undergoes a proteolytic processing event generating two subunits (DeJong and Roeder 1993; Ma et al. 1993; Yokomori et al. 1993), whereas in yeast, processing of the large subunit does not occur (Ranish et al. 1992). As a result, the endogenous TFIIA in higher eukaryotes is composed of three subunits in contrast to the two subunits found in yeast (Ranish and Hahn 1991; Ranish et al. 1992). However, thus far we have failed to detect any functional differences between the processed and unprocessed TFIIA. Instead, unprocessed dTFIIA-L efficiently interacts with dTFIIA-S, forming an active holoTFIIA (dTFIIA-L/S). Interestingly, holo-TFIIA is able to bind TBP with a much greater efficiency than either of the individual subunits. Moreover, binding of TBP to TATA box DNA was markedly stabilized in the presence of holo-TFIIA. Finally, holo-TFIIA can increase TFIIDdirected basal transcription as well as activation by some sequence-specific regulatory factors. Thus, it appears that the unprocessed TFIIA performs all the functions attributable to endogenous processed TFIIA (Reinberg et al. 1987; Ranish and Hahn 1991; Cortes et al. 1992). It remains unclear, however, why the processing of the large subunit occurs among the higher eukaryotes but not in yeast. It is conceivable that the processed form functions more efficiently in vivo or, alternatively, is only required under certain situations not yet determined.

How does TFIIA enhance transcription? In earlier studies it was proposed that TFIIA might counteract the effects of negative cofactors, suggesting that TFIIA may function more as an antirepressor than as an enhancer of basal transcription (Cortes et al. 1992; Merino et al. 1993|. However, it is unlikely that TFIIA can be delegated merely to such a passive role. For example, an early study using crude preparations of TFIIA reported that it can aid the binding of TBP to a wide spectrum of promoter sequences (Lee et al. 1992). Similarly, our footprinting data demonstrated that purified holo-TFIIA may play an important role in recruiting and stabilizing TBP on DNA even in the absence of negative regulators. Therefore, basal transcription may be enhanced by TFIIA simply by facilitating the recruitment of TBP and/or TFIID to the template. This idea is supported by our observation that TFIIA stimulates basal transcription by TBP, especially when low concentrations of TBP are used (data not shown). However, in vivo, TBP exists predominantly as part of the TFIID complex that consists of TAFs and other cofactors, including, potentially, both positive and negative effectors (Pugh and Tjian 1990; Dynlacht et al. 1991; Meisterernst and Roeder 1991; Meisterernst et al. 1991). It is therefore possible that these other cofactors influence the role of TFIIA. For instance, negative cofactors such as DR1, DR2, or components of USA (Meisterernst et al. 1991; Inostroza et al. 1992; Merino et al. 1993/ may inhibit basal transcription by destabilizing TFIID-DNA interactions. A simple mechanism for TFIIA action would be to compete with these negative cofactors for binding to TBP. It is also conceivable that TFIIA works by binding directly to cofactors. For example, we have shown that dTFIIA-L interacts selectively with some of the TAFs /Yokomori et al. 1993; K. Yokomori, unpubl.). Therefore, these protein-protein interactions may work in concert to modulate the formation of stable initiation complexes at the promoter and stimulate transcription. Presently, we cannot distinguish between these different alternative mechanisms, which may not be mutually exclusive.

An intriguing result we obtained was the apparent synergism between promoter-specific activators and TFIIA in directing transcriptional activation in vitro. In the case of the chimeric activator GAL-VP16-C, we observed virtually no activation in the absence of TFIIA but efficient activation in the presence of TFIIA. This suggests a potentially critical role of TFIIA in modulating transcriptional activation, at least under in vitro transcription conditions. The effect of TFIIA on activation was most dramatic with amounts of TFIIA that had little or no effect on basal transcription. Thus, we were able to discriminate between the effects of TFIIA on activation from those on basal transcription. It is not clear whether TFIIA acts via distinct or similar mechanisms to influ- 
ence basal versus activated transcription. In either case, it seems likely that holo-TFIIA can have a dramatic influence on the ability of certain activators to enhance transcription and therefore acts in a manner reminiscent of coactivators.

By what mechanism might TFIIA function to enhance activation? A simple possibility is that TFIIA interacts directly with activation domains, thus serving as an adaptor to link activators to the basal machinery. Interestingly, our preliminary studies indicate that dTFIIA indeed can bind directly to some activators (K. Yokomori and J.-L. Chen, unpubl.). A similar situation has been reported for the basal factor TFIIB, which interacts with the activation domains of the viral trans-activator VP16 (Lin and Green 1991). Alternatively, TFIIA may function as part of the TBP-TAF complex in response to activators, possibly participating in a subsequent step in the process of activation. It will be instructive to test the function of TFIIA at different stages of the transcription cycle to gain further insight concerning its possible mode of action.

In the course of our studies, we noticed several important parameters that can influence the activity of TFIIA. First, the requirement for TFIIA during transcription seems to vary between different activators, suggesting that its mechanism of activation may be activator-specific. Second, our unexpected results with Zta suggest that some activators may be able to bypass the TFIIA requirement, at least under certain conditions. This result was unexpected because recent studies reported the likely involvement of TFIIA during activation by Zta (Chi and Carey 1993; Lieberman and Berk 1994). Our footprint experiments suggest that TFIIA may selectively influence the binding of TBP and, presumably, TFIID to certain DNA templates. Therefore, the function of TFIIA may also be affected by core promoter sequences. Among the three promoters tested in our study, the AdML promoter showed the most striking TFIIA dependence during transcriptional activation. A similar effect was reported in the case of the repressor DR2 whose function may also depend on the context of different promoters (Merino et al. 1993). Whatever the mechanism, our findings establish that TFIIA is an important component of the initiation complex that will probably play a role in transcriptional regulation, possibly as a result of multiple events and pathways. Recently, our laboratory has succeeded in reconstituting activator-dependent transcription using recombinant TBP-TAF subunits (Chen et al. 1994) With the availibility now of recombinant TFIIA, it should eventually be possible to study the fine-tuning mechanisms of transcriptional activity by TFIIA by using a defined transcription system.

\section{Materials and methods}

Purification of dTFIIA and peptide sequencing of the $14-k D$ protein

Preparation of nuclear extracts from Drosophila embryos were essentially as described previously (Dynlacht et al. 1989; Wampler et al. 1990). TFIIA was either immunoprecipitated directly from PVA extracts using affinity-purified polyclonal antibodies against dTFIIA-L (Yokomori et al. 1993) or obtained from $0.5 \mathrm{M}$ eluate of the immunopurified TFIID as described previously (Yokomori et al. 1993). TFIIA was precipitated in 25\% TCA containing $1 \mathrm{mg} / \mathrm{ml}$ of deoxycholate. The TCA precipitate was resolved by SDS-PAGE, transferred to PVDF membrane (Millipore), and stained with Ponceau S. Proteins in the $14-\mathrm{kD}$ size range were excised from the membrane, digested with LysC protease, fractionated by reverse-phase HPLC, and subjected to microsequencing.

Cloning of cDNAs for the small subunit of Drosophila TFIIA

The $\lambda$ cDNA clones isolated from an eye disc-specific library (A. Cowmam, unpubl.) were subcloned into pBluescript $\mathrm{KS} \mid+$ ). cDNA clones from a $\lambda$-ZAP embryonic library (M. Noll, unpubl.) were rescued as pBluescript SK - plasmids. The sequence was determined on both strands. Computer analysis was performed using the GCG FASTA and TFASTA programs to search SWISS-PROT, PIR, GenBank, and EMBL data bases.

Expression of recombinant $\mathrm{ATFIIA-S}$ and preparation of holo-TFIIA (L/S)

dTFIIA-S cDNA was subcloned into pAR3038 for E. coli T7 expression (Rosenberg et al. 1987). For radiolabeled protein used in coimmunoprecipitation, this construct was used directly for in vitro transcription-translation reactions (T7 TNT system, Promega). Protein was also expressed in E. coli (BL21/DE3). After induction with IPTG, cells were harvested and then resuspended in HEMG (0.1) buffer [25 mM HEPES at pH 7.5, $12.5 \mathrm{~mm}$ $\mathrm{MgCl}_{2}, 0.1 \mathrm{mM}$ EDTA, $10 \%$ (vol/vol) glycerol] containing $0.1 \mathrm{M}$ $\mathrm{KCl}, 0.1 \% \mathrm{NP}-40,1 \mathrm{mM}$ DTT, and protease inhibitor PMSF. After sonication and centrifugation, dTFIIA-S protein was exclusively in the insoluble fraction, which was further washed by HEMG containing $0.25 \mathrm{M} \mathrm{KCl}$ to get rid of other bacterial proteins. Protocols for denaturation and renaturation of the proteins were essentially as described (Ranish et al. 1992). For both dTFIIA-L (Yokomori et al. 1993) and dTFIIA-S, $3 \mathrm{mg}$ of total protein was denatured in $7 \mathrm{M}$ Urea for $2 \mathrm{hr}$ at $4^{\circ} \mathrm{C}$. After centrifugation, the supernatants were either renatured separately or mixed and renatured by dialysis in HEMG containing $0.15 \mathrm{KCl}$ overnight. The protein concentration of soluble materials were analyzed either by Bio-Rad or Coomassie staining after SDSPAGE (see Fig. 3A). This material was used in the DNA-binding and transcription experiments.

cDNA was also subcloned into pVL1393 (Pharmingen) and pVL1392-HA (S. Ruppert, unpubl.) for expression of dTFIIA-S and HA-tagged dTFIIA-S in the baculovirus system using Sf9 cells.

\section{Generation of polyclonal antibodies against dTFIIA-S}

The procedure was as described previously /Yokomori et al. 1993). Bacterially expressed insoluble dTFIIA-S described above was resolved by SDS-PAGE, and the overexpressed protein was excised from the gel after staining with Coomassie blue. The gel slices were crushed and mixed with Freund's complete or incomplete adjuvant and injected into rabbits subcutaneously. Specificity of antisera to the recombinant protein was analyzed by Western blot.

\section{Protein-protein interaction assays}

In immunoprecipitation-Western blot assays, the PVA extract was immunoprecipitated by either anti-dTFIIA-L antibodies or 
anti-dTAF $\mathrm{TI}_{\mathrm{II}} 250$ monoclonal antibody preincubated with protein A-Sepharose beads (Pharmacia). Beads were washed thoroughly with HEMG $(0.1)$ containing $0.1 \%$ NP- $40,1 \mathrm{mM}$ DTT, and 0.2 mM protease inhibitor AEBSF (Calbiochem). The proteins on the beads were denatured with $2 \times$ sample buffer [ $10 \%$ glycerol, 0.7 M $\beta$-mercaptoethanol, $3 \%$ SDS, and $0.5 \times$ upper Tris buffer $l \mathrm{pH}$ $6.81]$ and resolved by SDS-PAGE. Proteins were then transferred to nitrocellulose membrane and analyzed by Western blot using anti-dTFIIA-S polyclonal antisera. In coimmunoprecipitation assays, the primary protein in a crude extract from baculovirusinfected Sf9 cells (e.g., dTBP, HA-dTFIIA-L, or HA-dTFIIA-S) $(1-2 \mu \mathrm{g})$ was incubated at $4^{\circ} \mathrm{C}$ with protein $\mathrm{A}-$ Sepharose beads preincubated with anti-dTBP (R.O.J. Weinzierl, unpubl.) or antiHA peptide (Bafco). After $2 \mathrm{hr}$, the beads were washed and incubated further with the ${ }^{35} \mathrm{~S}$-labeled second protein (e.g., dTFIIA-L, dTFIIA-LN, dTFIIA-LC, or dTFIIA-S) for $2.5 \mathrm{hr}$ at $4^{\circ} \mathrm{C}$. Beads were washed thoroughly, and the proteins on the beads were denatured and subjected to SDS-PAGE as described above. The signals were visualized by autoradiography.

\section{DNA-binding assays}

Mobility retardation assay was performed as described previously (Yokomori et al. 1993), using ${ }^{32}$ P-labeled synthetic oligonucleotides containing a TATA element. Baculovirus-expressed dTBP ( $\sim 30$ ng per reaction) was kindly provided by B. Dynlact (Dynlacht et al. 1993). A portion of the $0.5 \mathrm{M}$ eluate ( $\sim 20 \mathrm{ng}$ of total protein per reaction/ was used in the presence of dTBP as a positive control for TBP-TFIIA complex on DNA /Yokomori et al. 1993). Bacterially produced L/S, L, or S $/ \sim 10 \mathrm{ng}$ of total protein per reaction/ was mixed with a probe in the presence or absence of dTBP. The mixture was incubated for $30 \mathrm{~min}$ at room temperature and resolved on $5 \%$ acrylamide gel containing 0.5 M Tris-glycine buffer, $0.02 \% \mathrm{NP}-40$, and $1 \mathrm{~mm}$ EDTA.

DNase I footprinting assay was performed according to the procedure described previously (Hoey et al. 1990), using bacterially produced dTBP and dTFIIA-L, dTFIIA-S, or dTFIIA-L/S described above. Preparation of dTBP is essentially as described (Hoey et al. 1990).

\section{In vitro transcription}

Transcription assay was done essentially as described (Dignam et al. 1983), using a fractionated HeLa transcription system, including a fraction containing all the general factors except TFIIA and TFIID (phosphocellulose, $0.5 \mathrm{M}$ eluate) and a hTFIID fraction (phosphocellulose, $1.0 \mathrm{~m}$ eluate). Instead of the hTFIIA fraction (phosphocellulose $0.1 \mathrm{M}$ flowthrough), bacterially expressed $L / S, L$, or $S$ was used in various concentrations /total protein concentration of $\sim 1-30 \mathrm{ng}$ ). Transcription reactions were performed in a final volume of $25 \mu \mathrm{l}$ in buffer containing $40 \mathrm{~mm}$ HEMG, $4 \mathrm{~mm}$ spermidine, and $100 \mathrm{ng}$ of DNA template. The reaction mixture was incubated for $30 \mathrm{~min}$ at $30^{\circ} \mathrm{C}$. A final concentration of $0.5 \mathrm{~mm}$ rNTPs were then added and incubated for an additional $15 \mathrm{~min}$ at $30^{\circ} \mathrm{C}$. The products were detected by primer extension using ${ }^{32} \mathrm{P}$-labeled primers and analyzed by denaturing polyacrylamide gel electrophoresis. The DNA templates used include $\mathrm{GAL}_{5} \mathrm{AdML}-\mathrm{TdT}$, in which the AdML TATA box was fused to TdT initiator and CAT gene (E. Pascal, unpubl.), GAL ${ }_{5} \mathrm{E} 4 \mathrm{~T}$ (Lin et al. 1988), GC $\mathrm{G}_{3} \mathrm{E} 1 \mathrm{BCAT}$ (Dynlacht et al. 1991), and $Z_{7}$ E4TCAT (Carey et al. 1992). Signals were visualized by autoradiography and quantitated by PhosphorImager analysis (Molecular Dynamics).

\section{Acknowledgments}

We thank Danny Reinberg for providing sequence information of the small subunit of human TFIIA before publication. Sharleen Xiaying Zhou performed peptide sequencing of the 14$\mathrm{kD}$ subunit of dTFIIA. We are grateful to Paul Lieberman for kindly providing $Z_{\text {ta }}$ protein and $\mathrm{Z}_{7} \mathrm{E} 4 \mathrm{TCAT}$ DNA template and Steve Hahn for informing us of the presence of the rice homolog. We thank Robert Weinzierl for the monoclonal antibody specific for $\mathrm{dTAF}_{\mathrm{II}} 250$, Jim Goodrich for providing GAL4VP16 protein, Cathy Thut for GAL4-VC protein, and Edith Wang for various reagents and technical suggestions for in vitro transcription. We also thank Grace Gill, Jim Goodrich, Edith Wang, and Stig Hansen for critical reading of the manuscript. K.Y. is supported by a Leukemia Society fellowship and C.P.V. by a long-term European Molecular Biology Organization fellowship. This work was supported in part by a grant from the National Institutes of Health to R.T.

The publication costs of this article were defrayed in part by payment of page charges. This article must therefore be hereby marked "advertisement" in accordance with 18 USC section 1734 solely to indicate this fact.

\section{References}

Buratowski, S., S. Hahn, L. Guarente, and P.A. Sharp. 1989. Five intermediate complexes in transcription initiation by RNA polymerase II. Cell 56: 549-561.

Carey, M., J. Kolman, D.A. Katz, L. Gradoville, L. Barberis, and G. Miller. 1992. Transcriptional synergy by the Epstein-Barr virus transactivator Zebra. J. Virol. 66: 4803-4813.

Chen, J.-L., L.D. Attardi, C.P. Verrijzer, K. Yokomori, and R. Tjian. 1994. Assembly of recombinant TFIID reveals differential coactivator requirements for distinct transcriptional activators. Cell (in press).

Chi, T. and M. Carey. 1993. The ZEBRA activation domain: Modular organization and mechanism of action. Mol. Cell. Biol. 13: 7045-7055.

Cortes, P., O. Flores, and D. Reiberg. 1992. Factors involved in transcription by mammalian RNA polymerase II: Purification and analysis of transcription factor IIA and identification of transcription factor IIJ. Mol. Cell. Biol. 12: 413-421.

DeJong, J. and G. Roeder. 1993. A single cDNA, hTFIIA/ $\alpha$, encodes both the p35 and p19 subunits of human TFIIA. Genes \& Dev. 7: 2220-2234.

Dignam, J.D., P.L. Martin, B.S. Shastry, and R.G. Roeder. 1983. Eukaryotic gene transcription with purified components. Methods Enzymol. 101: 582-598.

Dynlacht, B.D., L.D. Attardi, A. Admon, M. Freeman, and R. Tjian. 1989. Functional analysis of NTF-1, a developmentally regulated Drosophila transcription factor that binds neuronal cis elements. Genes \& Dev. 3: 1677-1688.

Dynlacht, B.D., T. Hoey, and R. Tjian. 1991. Isolation of coactivators associated with the TATA-binding protein that mediate transcriptional activation. Cell 55: 563-576.

Dynlacht, B.D., R.O.J. Weinzierl, A. Adman, and R. Tjian. 1993. The dTAFII80 subunit of Drosophila TFIID contains betatransducin repeats. Nature 363: 176-179.

Hoey, T., B.D. Dynlacht, M.G. Peterson, B.F. Pugh, and R. Tjian. 1990. Isolation and characterization of the Drosophila gene encoding the TATA box binding protein, TFIID. Cell 61: $1179-1186$.

Inostroza, J.A., F.H. Mermelstein, I. Ha, W.S. Lane, and D. Reinberg. 1992. Dr1, a TATA-binding protein-associated phosphoprotein and inhibitor of class II gene transcription. Cell 70: $477-489$.

Lee, D.K., J. DeJong, S. Hashimoto, M. Horikoshi, and R.G. Roeder. 1992. TFIIA induces conformational changes in 
TFIID via interactions with the basic repeat. Mol. Cell. Biol. 12: 5189-5196.

Lieberman, P.M. and A.J. Berk. 1994. A mechanism for TAFs in transcriptional activation: Activation domain enhancement of TFIID-TFIIA-promoter DNA complex formation. Genes \& Dev. 8: 995-1006.

Lin, Y.-S., M.F. Carey, M. Ptashne, and M.R. Green. 1988. GAL4 derivatives function alone and synergistically with mammalian activators in vitro. Cell 54: 659-664.

Lin, Y.-S. and M.R. Green. 1991. Mechanism of action of an acidic transcriptional activator in vitro. Cell 64: 971-981.

Ma, D., H. Watanabe, F. Mermelstein, A. Admon, K. Oguri, X. Sun, T. Wada, T. Imai, T. Shiroya, D. Reinberg, and H. Handa. 1993. Isolation of a cDNA encoding the largest subunit of TFIIA reveals functions important for activated transcription. Genes \& Dev. 7: 2246-2257.

Meisterernst, M. and R.G. Roeder. 1991. Family of proteins that interact with TFIID and regulate promoter activity. Cell 67: 557-567.

Meisterernst, M., A.L. Roy, H.L. Lieu, and R.G. Roeder. 1991. Activation of class II gene transcription by regulatory factors is potentiated by a novel activity. Cell 66: 981-994.

Merino, A., K.R. Madden, W.S. Lane, J.J. Champoux, and D. Reinberg. 1993. DNA topoisomerase I is involved in both repression and activation of transcription. Nature 365: 227232.

Pugh, B.F. and R. Tjian. 1990. Mechanism of transcriptional activation by Spl: Evidence for co-activators. Cell 61: 11871197.

Ranish, J.A. and S. Hahn. 1991. The yeast general transcription factor TFIIA is composed of two polypeptide subunits. /. Biol. Chem. 266: 19320-19327.

Ranish, J.A., W.S. Lane, and S. Hahn. 1992. Isolation of two genes that encode subunits of the yeast transcription factor IIA. Science 255: 1127-1129.

Reinberg, D., M. Horikoshi, and R. Roeder. 1987. Factors involved in specific transcription in mammalian RNA polymerase II. Functional analysis of initiation factors IIA and IID and identification of a new factor operating at sequences downstream of the initiation site. J. Biol. Chem. 262: 33223330 .

Rosenberg, A.H., B.N. Lade, D.S. Chui, S.W. Lin, J.J. Dunn, and F.W. Studier. 1987. Vectors for selective expression of cloned DNAs by T7 RNA polymerase. Gene 56: 125-135.

Samuels, M., A. Fire, and P.A. Sharp. 1982. Separation and characterization of factors mediating accurate transcription by RNA polymerase II. J. Biol. Chem. 257: 14419-14427.

Van Dyke, M.W., R.G. Roeder, and M. Sawadogo. 1988. Physical analysis of transcription preinitiation complex assembly on a class II gene promoter. Science 241: 1335-1338.

Wampler, S.L., C.M. Tyree, and J.T. Kadonaga. 1990. Fractionation of the general RNA polymerase II transcription factors from Drosophila embryos. I. Biol. Chem. 265: 21223-21231.

Wang, W., J.D. Gralla, and M. Carey. 1992. The acidic activator GAL4-AH can stimulate polymerase II transcription by promoting assembly of a closed complex requiring TFIID and TFIIA. Genes \& Dev. 6: 1716-1727.

Yokomori, K., A. Admon, J.A. Goodrich, J.-L. Chen, and R. Tjian. 1993. Drosophila TFIIA-L is processed into two subunits that are associated with the TBP/TAF complex. Genes \& Dev. 7: 2235-2245. 


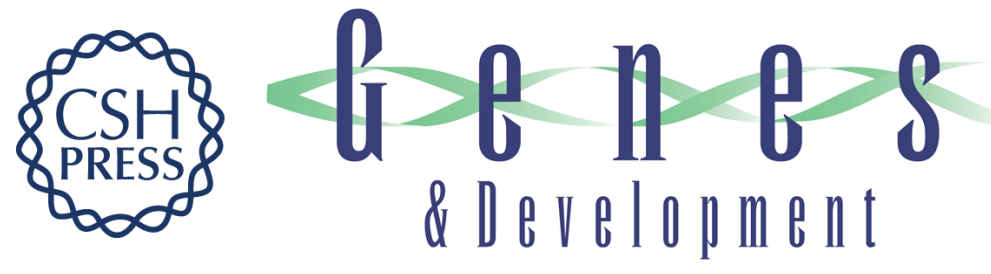

\section{Drosophila TFIIA directs cooperative DNA binding with TBP and mediates transcriptional activation.}

K Yokomori, M P Zeidler, J L Chen, et al.

Genes Dev. 1994, 8:

Access the most recent version at doi:10.1101/gad.8.19.2313

References This article cites 29 articles, 16 of which can be accessed free at:

http://genesdev.cshlp.org/content/8/19/2313.full.html\#ref-list-1

License

Email Alerting

Service

Receive free email alerts when new articles cite this article - sign up in the box at the top right corner of the article or click here.

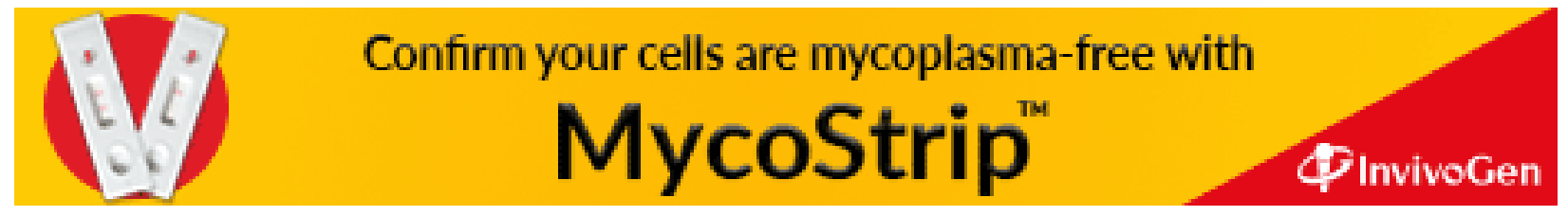

